\title{
Predictors of Sexual Scripts Among Young, Sexually-Active, Substance- Using African American Women
}

Mandy J Hill*, Misha Granado, Yolanda Villarreal, Jamie Fuega, David J Robinson and Angela L Stotts

Department of Emergency Medicine, McGovern Medical School at UT Health Science Center at Houston, Texas, USA

\begin{abstract}
Background: The HIV epidemic in the US continues to disproportionately affect the health of young, African American women. The focus here is on predictors of sexual scripts, which are roadmaps to sexual decision making. The objective is to examine life experiences, normative beliefs and cultural predictors of sexual scripts that place young, sexually-active, substance-using, African American women (YSSAAW), a population with significant vulnerability to HIV, at even greater risk of becoming HIV positive.
\end{abstract}

Methods: Face-to-face, tablet-assisted, semi-structured interviews were conducted with 30 YSSAAW in a private or public emergency department in Houston, TX, USA. Interviews were professionally transcribed, then coded by a trained 3-member coding team. One interview was used to create the codebook. Codes were organized into primary themes during face-to-face meetings. Inter-coder reliability was assessed and confirmed using Cohen's Kappa statistics, demonstrating a nearly perfect agreement between coders 1 and $2(K=0.93)$.

Results: Three primary themes were described as predictors of sexual scripts: emotional wounds, norms and decision making. Prevalent codes among YSSAAW within the emotional wounds theme included infidelity (43.33\%) and parental dynamics (56.67\%). Under the norms theme, we found $66.7 \%$ of YSSAAW discussed their communication norms and $30 \%$ disclosed cultural norms. Within the sexual decision making theme, we gained relevant information and implications on relationship longevity and having an STI history among $46.67 \%$ of the sample.

Conclusion: Primary predictors of high risk sex revealed sexual scripts that demonstrated gender-based power differentials; thereby, supporting utility of a theoretical framework that includes the Sexual Script Theory and the Theory of Gender and Power. The logic model illustrates how emotional wounds from life experiences (i.e., trauma, abuse, abandonment) and socially acceptable norms establishes the sexual script of YSSAAW; whereby, high risk sex is the most likely outcome relative to prevention strategies.

Keywords: Substance use; Sexual decision making; Sexual scripts; HIV prevention; Young adults; African American women; Emergency department

\section{Introduction}

HIV remains a major public health problem for African American (AA) women in the United States (US) [1] who are disproportionately affected by this disease. The Center for Disease Control and Prevention (2016) reports, of women living with HIV, 61\% are AA as compared to $17 \%$ of Hispanic and non-Hispanic White women [2]. Heterosexual AA women have consistently been the fourth leading risk group in new HIV infections and young AA women, under the age of 25, are three times more likely to be infected with HIV as compared to young women of other ethnic and racial groups. A plausible contributor to this increased incidence is the high prevalence of substance use among AA women. In general, substance use exacerbates vulnerability to HIV among young, sexually-active, substance-using AA women (YSSAAW) and HIV risks among YSSAAW exceed risks among AA women. Substance use has been linked to low perceived vulnerability to HIV infection, which has been shown to increase the chance that a woman will engage in risky sex behaviors (i.e., condomless sex) [3]. Our local ED-based HIV testing program reported HIV incidence of $0.6 \%$ [4]. Houston substance use prevalence rates of injection drug use is $13.4 \%$ and non-injection drug use is $32.1 \%$ [5]. The imposed burden of HIV to YSSAAW is evident and this population would benefit from intervention.

Interventions aimed to reduce HIV risk, inclusive of counseling and education on best sex practices, are warranted and necessary with YSSAAW [6]. Emergency Department (ED) populations report high STI rates nationally [7-12]; thus, identification of YSSAAW who are seeking care for non-emergent conditions in the ED affords the necessary access and sufficient time to administer rapid and adaptive HIV risk reduction interventions to a population with significant vulnerability to HIV infection and limited access to preventive or routine care $[13,14]$.

Several HIV/STI behavioral interventions with AA women in the US have shown efficacy and recommend gender and cultural adaptation $[15,16]$; this is especially true for subgroups of women at increased risk for HIV. Moreover, adapting interventions to varied settings such as the ED where [16] vulnerable, substance-using populations at especially high risk for HIV infection can be accessed, are especially warranted. ED-based interventions have demonstrated efficacy for separately addressing HIV prevention for people living with HIV [17], people at risk for HIV [18-28] and substance users [29]. However, a dearth of interventions remains for substance users at-risk for HIV who obtain non-emergent care in the ED. Findings from a clinic-based randomized controlled trial, the Girls OnGuard study, a study focused on addressing

*Corresponding author: Mandy J Hill, DrPH, MPH, Assistant Professor McGovern Medical School at UT Health Science Center at Houston, Department of Emergency Medicine, 6431 Fannin St., JJL 420, Houston, TX 77030, USA, Tel: 713-500-7661; Fax: 713-500-7884; E-mail: Mandy.J.Hill@uth.tmc.edu

Received December 23, 2016; Accepted January 14, 2017; Published January 21,2017

Citation: Hill MJ, Granado M, Villarreal Y, Fuega J, Robinson DJ, et al. (2017) Predictors of Sexual Scripts Among Young, Sexually-Active, Substance-Using African American Women. JAIDS Clin Res 8: 655. doi: 10.4172/2155-6113.1000655

Copyright: (c) 2017 Hill MJ, et al. This is an open-access article distributed under the terms of the Creative Commons Attribution License, which permits unrestricted use, distribution, and reproduction in any medium, provided the original author and source are credited. 
STI prevention among AA adolescent women 13-18 years of age, suggests that brief clinic-based health promotion interventions can be done without interrupting clinic operations, procedures and patient flow. Findings of clinic-based HIV/STI prevention studies present important considerations for ED-based interventions, as strategies that prevent interruption of clinical care in other settings is applicable to the ED setting [30]. Our research team initiated and completed formative work establishing feasibility of interventions to minimize HIV/STI infections in ED settings among AA women [31-35]. The research presented in this paper was the next step on the continuum towards implementation of an adaptation to an evidence-based intervention for HIV prevention in the ED. Specifically, we aim to identify predictors of sexual scripts that determine whether YSSAAW make sexual decisions to either engage in high risk sex or preventive behaviors.

HIV/substance-abuse (SA) prevention interventions have shown efficacy among AA women. Concurrently, interventions targeting gender-based power imbalances that contribute to high risk sex behaviors among YSSAAW are lacking among existing HIV interventions. The Sexual Script Theory (SST) [36-42] and the Theory of Gender and Power (TGP) [41,43-48] offer a theoretical framework to effectively adapt interventions to be culturally relevant to YSSAAW. Stephens and Phillips' focus of females' sexual script development research with AA women explores ways culture shapes perception, expression of appropriate and socially acceptable sexual behavior, individual level expectations, and experiences of behaviors that occur in series [49] within a population at high risk for HIV [36,50-60]. The SST articulates the way sexual scripts provide instructions (i.e., a roadmap) for clarifying the intention and meaning of sexual cues and responding to them, which in turn informs how men and women behave in sexual situations [50,51,60-63]. Sexual scripts in Western culture sometimes support women in heterosexual relationships to defer to the male partner for sexual decision making, resulting in situations where women are vulnerable to the decisions made by their male counterparts $[51,63,64]$

This, in turn, leads us to the TGP, which explores how gender-based power imbalances strongly influence the sexual behaviors that place AA women at high risk for HIV and constrain a woman's negotiation ability [65]. Influence and constraint are compounded by feelings of powerlessness and meekness [43]. Wingood led the focus of Connely's TGP on powerlessness of AA women in sexual decision making [44-46,66]. Within sexual scripts of the AA women, there is a social standard where the power in the sexual relationship favors the male and inconsistent condom use is attributed to low sexual relationship power [16,49,51,53,59,60,65,67-71], supporting a dynamic where the female is vulnerable to decisions made by her partner [51,64]. By making concerted efforts to better understand these social standards, researchers are better equipped to recognize diverse effects of social support and the vital role it plays in conceptualizing the influential power of the male gender on sexual risk among AA women [59,63].

SST and TGP demonstrate the influence of societal factors and gender roles on sexual behaviors among AA women [37,38,45,46,49]. To date, these concepts have not been considered in the guiding theoretical framework of HIV prevention interventions with YSSAAW [72]. Historically, researchers have explored varied types of sexual scripts [49,54,55,57,59,72-77]. Most recently, US-based research related to sexual scripts explored gender and sexual behavior among adolescents and college students [78,79], stigma associated with being sexually inexperienced [80], sexual scripts that tolerate sexual coercion among college students and nonconsensual sex/rape among AA and White adolescent and college women [81-83], hip hop media as a powerful mechanism promoting saliency of sexual scripts for AA women [84] and most related to this study was a qualitative study assessing the effects of sexual victimization history of undergraduate women on hypothetical sexual script characteristics [85]. To our knowledge, this is the first publication to explore predictors of sexual scripts, particularly among a population with significant HIV risks.

In laymen's terms, sexual scripts are an individual's instruction manual to sexual decision making. In order to grasps factors that influence the development of sexual scripts for YSSAAW, we must explore cultural, societal and life experiences that contribute to the development of what are normal to them, specifically normal sexual behaviors. Cultural esteem gained by maintaining a heterosexual relationship with a man within the AA community is highly valued and often leads women to focus on benefits of relationships (i.e., stability, finances, family) and to ignore costs by male partners that often include infidelity [86], concurrency that is rarely discussed within partnerships, and interference with safer sexual decisions stemming from frequent alcohol and substance use [87]. Specifically, sexual scripts of YSSAAW are more likely to involve riskier sexual behaviors than scripts of other/ older AA women. YSSAAW are more likely to be victims of rape, physical violence and stalking by an intimate partner [72,88]; thus, their sexual scripts are often closely aligned with victimization and powerlessness.

The combined implications of societal and culture influences with a personal history of sexual violence makes YSSAAW an especially vulnerable population warranting the attention of HIV and STI interventionists. Strategies to address HIV risk among this subgroup of AA women are timely and warranted. Gender-power differentials within a substance-using context results in increased risk HIV/AIDS and other STIs, as well as sexual and physical violence [88]. These heightened risks may be attributed to social norms associated with some substance-using cultures, such as transactional sex and/or sexual availability perceived as promiscuous, both of which place women at greater risk for sexual violence [89]. Research has found $70 \%$ of women being treated for drug abuse report histories of physical and sexual abuse, with an onset prior to 11 years of age coupled with repeat occurrences. Others have found that $32 \%$ of young women who became pregnant $<18$ years had a history of rape or incest reported drug use (i.e., crack, cocaine, other drugs) (NIDA, 2002). Of these women, the majority reported having low self-esteem, and engaged in more delinquent behaviors than women without a history of sexual violence [88].

This subgroup of AA women has distinctive characteristics that are not adequately addressed in extant interventions. Adaptation of interventions for this highly individualized subgroup of young women at significant risk of HIV infection, utilizing a gender and power theoretical lens, is lacking and warranted. Moreover, adaptation of evidence based interventions for specific population is most effective when informed by stakeholders within the subgroup [90]. Thus, this study contributes to the body of literature by examining and reporting predictors of sexual scripts among YSSAAW; a rare cross section of a community, seeking care in an ED setting in Houston, a major US metropolitan city.

\section{Purpose}

The purpose of this exploratory study was to identify predictors of sexual scripts that align with lifetime decision to engage in high risk sex among minority women with significant vulnerability to HIV - YSSAAW. 
Predictors of those scripts are analyzed and informed by the SST and the TGP, two theories that align well with the social and cultural norms of this population. Study findings contribute to literary evidence supporting the need to include a theoretical framework that addresses sexual scripts and gender-power differentials for use within adaptations of existing evidence based, HIV prevention interventions [72].

\section{Methods}

This exploratory study used a descriptive qualitative design. Researchers who are credentialed to do research at both hospitals used the hospital's electronic medical record system to identify eligible patients seeking care in the ED. We collected basic demographic data, including age, marital status, race/ethnicity, chief complaint, and social histories, including substance use (type of substance and frequency of use) and sexual activity (currently active $(\mathrm{Y} / \mathrm{N})$; number of partners) on all patients through the screening process of determining eligibility. This de-identified information was entered into Microsoft Excel. Participants completed a 20-40 min interview using an adapted, validated in-depth interview tool [91]. All questions posed were related to either the SST or the TGP. Topic foci were feelings about sex, descriptions on the development of sexual intimacy, detailed description of subject's first penetrative intercourse experience, gender differences, details on sexual history, knowledge of partner's sexual activities, sexual risk taking, and protective practices. Interviews were semi-structured; thus, a bank of questions from a validated instrument were asked, but the same questions were not asked in every interview (see interview guide in Appendix 1). All interviews started with the initial question, 'What does sex mean to you?' Interviewee responses were the primary influence to the direction of questions asked in each interview. Interviews were fluid and were tailored to the subject's comfort level on varied sensitive topics.

Semi-structured, in-depth interviews were conducted in private rooms within a private or public emergency department over a seven month period (September 2015-April 2016) to a convenience sample of 30 AA women aged 18-29 years, who presented to either a public $(n=15)$ or private $\mathrm{ED}(\mathrm{n}=15)$ for a non-emergent condition. These women were approached by a research associate or the study principal investigator and were asked to participate in the study. Written informed consent was obtained. The study was approved by the institutional review board (HSC-MS-14-0819). Interviews were tailored to subject' responses and were administered in a way to discover factors that contribute to either high risk sex or prevention behaviors. Study subjects were allowed to comment on both their personal experiences and the experiences of their peers. Interview questions were viewed and recorded by the interviewer on an iPAD device. Each study subject received a $\$ 25$ gift card at the conclusion of the interview for their participation. Clinical care was not interrupted for any study subjects.

\section{Data Analysis}

Interviews were audiotaped using an $\mathrm{iPAD}$ application (Voice Recorder by TapMedia Ltd v2.1) with subject's permission and consent. Field notes were taken and content was coded independently by each interviewer. Units of analysis were based on the words and colloquialism used by study subjects. The PI outsourced transcription of interviews to professional transcribers. Accuracy of transcriptions was verified by comparing the taped audio to the typed version. Transcribed audio were analyzed to categorize and identify relevant codes and themes using content analysis. A trained coding team $(n=3)$ coded quotes from 1 interview to create the codebook. All 30 transcripts were coded by the team. Codes were organized into themes by the group during face-to-face meetings. Codes were collapsed into themes informed by Wingood's focus of Connell's TGP on the powerless of AA women in sexual decision making [44-46,66] and Stephen and Phillip focus on female's sexual script development research of AA women [36,50-60]. The research team then conducted a frequency analysis on emerging themes using NVIVO11 and Microsoft Excel software. Inter-coder reliability was assessed and confirmed using IBM SPSS Statistics 24 to calculate Cohen's Kappa statistics. Agreement between coders 1 and 2 $(\mathrm{K}=0.93)$ showed nearly perfect agreement, coders 2 and $3(\mathrm{~K}=0.304)$ and coders 1 and 3 ( $K=0.24)$ had fair agreement [92].

\section{Results}

Basic demographic information was collected on enrolled subjects illustrating a relatively even distribution of age, marital status, and chief complaint category across both study sites (Table 1). Most of the subjects $(86.67 \%)$ were ages $18-25$ years, single (90\%), and sought primary care in an ED setting $(76.67 \%)$.

\section{Themes}

Three primary themes were identified from the interviews as predictors of sexual scripts. The themes that predicted sexual script development according to the logic model identified (Figure 1) were: a) emotional wounds, b) norms, and c) decision making. Each of these predictive themes was explored and illustrations of each them are provided verbatim from the taped interview.

\begin{tabular}{|l|c|c|}
\hline Variables & \multicolumn{2}{|c|}{ Emergency Departments } \\
\hline Age & Private (n=15) & Public (n=15) \\
\hline $\mathbf{1 8 - 2 1}$ & 6 & 6 \\
\hline $\mathbf{2 2 - 2 5}$ & 7 & 7 \\
\hline $\mathbf{2 6 - 2 9}$ & 4 & 3 \\
\hline Marital Status & 13 & 14 \\
\hline Single & 1 & 1 \\
\hline Married & 1 & 0 \\
\hline Missing & & 15 \\
\hline Race/Ethnicity & 15 & 11 \\
\hline African American & & 1 \\
\hline Chief Complaint & 12 & 3 \\
\hline Primary Care & 0 & 0 \\
\hline Dental Care & 2 & \\
\hline Pregnancy Related & 1 & \\
\hline Type of Substance Use reported & & \\
\hline
\end{tabular}

Table 1: Sociodemographic profile of study participants.

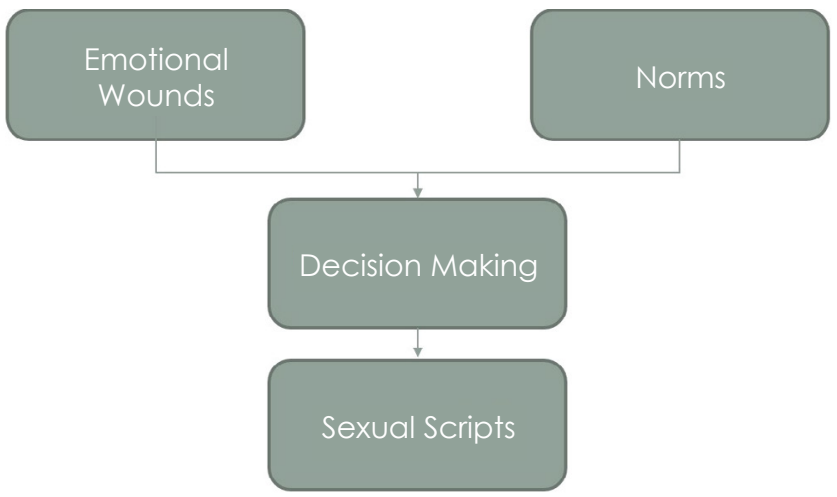

Figure 1: Thematic findings illustrating predictors of sexual scripts. 


\section{Emotional wounds}

YSSAAW participating in interviews frequently reported experiencing emotional trauma, heart ache and pain. The coding team described this theme as 'emotional wounds', a psychology-based term describing how the primitive development of sexual scripts supports condomless sex with main and casual partners, a script that begins early in life from the start of relationships with other people. Emotional wounds indicate an imbalance in our mental or physical world and is often a result of an experience separating us from what is generally considered good and necessary [93]. Interviews included sexual history; thus, descriptions of this historical information shed light on deeply seeded emotional wounds for some of the study subjects. Enrolled subjects described their emotional wounds as stemming from experiences that we coded $(n=22)$ as betrayal, infidelity, abandonment, regret after sex, rape, infidelity, race, pain and fear. Direct quotes from YSSAAW demonstrating infidelity and parental dynamics as sources for emotional wounds that remain years after the incident are described below.

Infidelity: Infidelity was referenced 19 times within 13 transcripts, indicating a prevalence of discussions on infidelity among $43.33 \%$ of YSSAAW enrolled. Within the emotional wounds theme, the code for infidelity was connected to hurtful life experiences with sexual partners. When discussing gender differences of men and women in their own age range, one participant described gender differences by stating,

'I think that men are more, more rules, have more rules than girls. Because it's like...they cheat everywhere. And like, there has not been a relationship where someone doesn't cheat on you in bed. Or have cheated on you. They cheat, like every relationship right now they cheat.'

When another subject was asked about her thoughts and feelings in relation to sexual interactions with her spouse, she said:

'I would - My husband used to cheat a lot so I would try to do different things to make him think that sex would make it better, change his cheating, try to do new things, but it never worked.'

Regarding infidelity, study subjects overall expressed polar opposite expectations regarding infidelity as an event that would either: 1) occur or 2) never occur. There were reports on a myriad of thoughts and feelings on infidelity ranging from distrust of a partner once trusted, strategies to prevent future infidelities, feelings of emotional disconnect following infidelity, future distrust to potential male partners based on historic experiences of infidelity by other men, to feelings of 'disgust so...' I left.

Parental dynamics: Parental dynamics was referenced 38 times within 17 transcripts, indicating parental dynamics may be predictive of sexual decisions and script adoptions among 56.67\% of YSSAAW. Parental dynamics described in this population revealed fear of YSSAAW at sexual debut, communication deficits between the subject and their parents during adolescents, a desire to please their parents, and absence of a fatherly presence. In one case, the interviewer inquired about the difference in concern about the mother finding about the sexual debut as opposed to the father. The subject's response was:

'Because I stay - My mom raised me, so I stay with her. My dad wasn't always in the picture.'

Another subject describes her penetrative sexual debut with a cousin and stated that this information was still unknown to her mother. Her response to the inquiry regarding her first penetrative sexual intercourse experience was,
'Well, he really didn't take my virginity... he took my butt. (laugh)...I was maybe like 6 or 7 . He was like 19 or 20, yes, he was older. And my mom, she still doesn't know, to this day'

Her response shed light on several codes, including rape, child abuse, parental dynamics, and the ability to dismiss the severity of the emotional wound imposed on her at the young age of 6 or 7 . In other cases related to parental dynamics, safety and willingness to be vulnerable and share sensitive information with the parent regarding the sexual debut experience was illustrated, as:

'I told her. We sat down like a month later and I told her...uh...she was just like what's wrong with you? She said she started seeing different habits or something. Or maybe she heard me over talking, and she was like do I think we need to have the talk again. Or whatever and I just went ahead and told her that it happened. And she was like "recently?" and I was like "yeah, recently," and I cried and...I just cried and she cried because it's like I wasn't her baby girl anymore. So...

\section{Norms}

Social norms that may contribute to sexual scripts of YSSAAW stem from codes $(n=11)$ identified under this theme, including normative communication styles, culture, peers, gender and relationship dynamics. Codes under the norms theme were routinely referenced among study subjects, as gender norms had 59 references within 21 sources accounting for $70 \%$ of subjects and communication norms had 56 references within 20 sources (66.67\% of subjects). Similarly, relationship dynamics, gender differences, relationship norms, communication norms and gender norms were represented by $66.67 \%$ of the sample, followed by peer norms representing $53.33 \%$ of the sample.

Communication norms: Dialogue coded as communication norms illustrated the mechanism of information sharing and depth of information shared between the subject and their sex partners, including comfort discussing prevention strategies with sex partners, sexual relationship that stemmed from friendships and willingness to express sexual desires with partners. One subject who expressed confidence when communicating her sexual desires to her partner stated:

'That comes with getting to know that person. Because I conversate a lot. So I'm going to, you know... before it even happens, you're going to know what I like, what I don't like and you know. Then when we experiment, then I can see if you do it right, and if not, I'm going to tell you this was not right, I didn't like the way this felt, let's try this and you know.'

Another subject expressed how her communication style changed after she was verbally or physically abused by former partners. She said:

'I guess the timing. Because when I first you know separated from my husband, I talked to people, but it's like I've been through so much with him, it takes one thing to trigger me and then I don't want to talk to you anymore. If you get loud with me, if you get aggressive with me, if you call me all kind of names-no, like I'm done. So it takes for you to mess up one time, you don't get that chance again because I let him mess up so much. So it's like with him, he loving, kind, caring, everything. It's like I don't deal with all of that, but if it ever came to that one time that he just was like disrespectful, that would be because I don't tolerate it anymore.'

Cultural norms: Although cultural norms were not coded as frequently as other codes under the norms theme (18 references of 9 
sources, $30 \%$ of YSSAAW), the coded data ties into other themes and codes that intersect with AA culture. For instance, one subject described the mindset of her peers where intermingling substance use and sex has become the norm and has a consuming effect on behavior. She said:

'But for my age group, we're very-they're very close minded. They don't want to expand their mind. The only thing they know is drink, smoke, sex. Drink, smoke, sex. It's all they know.

Another subject described the implications of being raised by a single mother with a history of failed marriages. Her description of family dynamics demonstrated the intersection of parental dynamics, cultural norms, gender norms and gender power differences as a cultural standard. Her remarks were,

'I was raised by a single mother. She's been married twice. My grandmother was married. Um, all the women in my family married, but we were also raised as women to be strong and independent. But to-what is the word I'm looking for? When it comes to looking for a husband, or being in a committed long term relationship, I don't want to say bow down to your man, but...To submit. Now there's women don't want to submit, that don't know how to let go when they find one, if they find one. Because most of them aren't looking, so they're hard and they're cold. They don't want to be dependent. We were taught to be independent, but to submit and let go when your man is there to lead in the right way. So yeah. It's-it's different and disappointing nowadays.'

\section{Decision making}

Following the influence of emotional wounds and norms on development of the sexual script, the decision making process is likely the final precursor to the script. Codes $(n=29)$ under the decision making theme included longevity, monogamy, best sex, STI history, spirituality and emotional connection.

Longevity: Longevity was coded 28 times among 14 (46.67\%) YSSAAW enrolled. Longevity related to the length of time the subject was acquainted with their former or current sexual partner prior to the sexual debut. The data revealed longevity correlated with long-term relationships, sharing a child, emotional connection, friendship, trust, loyalty, minimization of the sexual act and the ability to be manipulated and influenced by the male partner. When asked about the decision making process of moving from 'fear to have sex' to 'the decision to have sex', she responded:

'Just the person I was with at the time, I guess you could say he kind of persuade me to it. I was in a relationship with him for I guess two years before we actually... It was just you know feeling close to him and you know he kind of talked me into it. (laugh)'.

As in many cases, this subject minimized the sexual act and the manipulation that led up to it with laughter. We also explored the decision to engage in condomless sex with partners. When asked about consistent condom use with her partner, one subject stated:

'No we don't use condoms at all but if I'm dating you uh and it's for a short period of time we have to use condoms, but we've been dating for a while so no.'

In this response, we see a correlation between longevity and the sexual decision to engage in condomless sex (which was a separate code with 25 references from $56.67 \%$ of subjects [ $n=17]$ ), a correlation we found in several interviews.

An association was also found between perceived seriousness of a relationship, as an indicator of an established commitment, when relationships were long-term. One subject stated:

'Well, the relationship I'm in now, I feel that that is a serious relationship. We've been together for six years. We have three kids together. And there are a lot of things that we'll do for each other. He's there for me when I need him. When I'm feeling bad, when I'm feeling down he's there. He comforts me. He's always by my side.'

STI history: STI history was coded 17 times within 14 interview sources, indicating this history among $46.67 \%$ of YSSAAW enrolled. As part of a conversation about inconsistent condom use, subjects were asked about their decision to forgo condom use or use a condom. One subject's response was:

'Normally when I first started talking to someone, we always started off with protection. But along the way like if I develop a relationship and I feel like you there to stay around then I let my guard down like whatever. We don't have to use it because I feel like I'm with you, you with me and its okay. But it's not okay because dudes...they don't care about their body like women, and they don't go through stuff like we go through so they feel like they don't have signs of STDs or nothing like that, well I'm cool, but we can be over here cloudy-white, burning, all kinds of stuff. But that's the risk we have to take, we can be pregnancy, being irresponsible. It's like I know this stuff but I just don't...don't register it until I'm here, in a hospital or I can't take the burning or the itching, it's then, that's when it hit, like once they really working. That's when it hits me, and he out like I'm good what's wrong with you? ...Well I'm irritated and well I don't have no signs so if you go to the doctor wee-wee like if you - if...if something is wrong with you and they tell you then I know, then it's time for me to go, you know. But if I come back and tell you oh there aint nothing wrong with me and I'm over here burning to death, but if I tell you I'm good, oh you good, then you going to go on to the next one or you gonna try to come back to me and give it back to me. So stupid.

The influence of a historical STI on current and future sexual decision making was also explored. In some cases, an STI was the reason for several follow-up behaviors including a relationship ending, routine STI testing, monogamy by the subject in a current relationship, caution when establishing new relationships, and misnomers regarding their sexual health and vulnerability to future infections. In one case where the subject was asked about her perceived risk to HIV/STIs, she expressed,

'I think about it to the fullest extent, because honestly, I have attracted [contracted] chlamydia before. So I mean, I'm very open and exposed to HIV, any other STI and, um, I have a very weak immune system.'

\section{Discussion}

Several studies to date explored sexual scripts of AA women $[36,49,54,55,59,72,73,75,77,94]$. To our knowledge, this is the first study to move beyond the description of sexual scripts to identify and describe factors that are likely along the pathway to sexual script development among YSSAAW. This adds to a growing body of literature in HIV prevention field, as the decision tree of the sexual script can be interrupted and redirected when predictors of the sexual script of women with significant vulnerability to HIV (i.e., YSSAAW) are known. Cultural implications informing these predictors have the potential to be adequately addressed through HIV prevention intervention; thus, long-term intervention goals of designing and implementing tailored strategies to rewrite sexual scripts are made tangible.

As described, we conducted semi-structured, in-depth interviews 
during wait times of an ED visit of eligible YSSAAW receiving nonemergent health care to explore predictors of their normative decisions to engage in either high risk sex or to consistently utilize prevention measures (i.e., sexual scripts). Consistent with previous theoretical and empirical research on AA women and substance-using women, we found that the majority of women utilized sexual scripts that aligned with high risk sex $[59,70,73,75]$ as $56.67 \%$ reported adoption of sexual scripts that included condomless sex with sexual partners. Themes found reflect the major dimensions of sexual scripts identified by Bowleg et al. These scripts correlate with cultural values placed on maintaining a male partner and support the principles posed by Wingood et al. in the application of the TGP to cultural norms and sexual behaviors of AA women as it relates to perceived partner infidelity by the male partner [44-46,65,95-97]. These findings describe how sexual scripts are composed of gender-based power imbalances, cultural norms that support poor sexual decision making and a premise that supports low perceived vulnerability to HIV/STIs of women with significant vulnerability to such outcomes through minimization of the sexual act as a coping strategy. As such, these interview findings have important implications for revising and adapting HIV prevention interventions to improve cultural appropriateness for a population that could benefit from such changes, YSSAAW.

Unique to this sample of YSSAAW is the importance of outcomes and decisions stemming from emotional wounds that validate literary findings which repeatedly report that substance-using women are at heightened risk of HIV, STIs and sexual and physical violence, attributable to social norms aligning with substance use. Among YSSAAW enrolled, more than half (up to 56.67\%) reported experiencing emotional wounds at some point in their life. In a few cases, YSSAAW reported rape by trusted family members (i.e., cousins, fathers) at the vulnerable ages of 6-8 years. The profound effect of sexual violence occurring so early in the manifests in adulthood in several ways, including their sexual scripts. Research findings revealed that most women with a documented history of substance use report an early onset of physical and sexual abuse [88]. The combined effect of emotional wounds that were routinely ignored in the lives of these women, as evident by the frequent crying and breakdowns associated with discussing such hidden and secretive information during the interviews, coupled with substance use as a coping strategy and partnering with men who they often perceive as unfaithful $(43.33 \%$ reported perceptions of partner infidelity) creates a condition for significant vulnerability to HIV. Findings of this qualitative study further supports that emotional wounds contribute to sexual decisions to engage in high risk sex, particularly condomless sex with primary and/or casual partners.

It is noteworthy to discuss interview responses to inquiries regarding gender differences between men and women, as they indicated how perceptions of gender and power dictated sexual scripts and relationship expectations. As stated, partner infidelity was an expected outcome for $43.33 \%$ of YSSAAW enrolled and tolerance of this behavior was expressed as a cultural norm. To this point, strategies of the women to sustain the relationship when partner infidelity is known were described. Embedded in the AA culture is a hierarchal value system for women whereby their social status and worth is sometimes based on their ability to get a man and keep him. When an AA woman is successful at getting a man, the pathway to keeping him is often guided by matriarchs in the AA community that support women in the development and maintenance of sexual scripts that ignore poor behaviors of their men (i.e., infidelity, multiple sex partners, substance use) and focus on benefits of the relationship (i.e., stability, finances, family). This is coined the $80: 20$ rule, a theory that in a fairly healthy relationship, you only get $80 \%$ of what you need in a mate and the missing $20 \%$ represents wants that can be dismissed [86]. The social risk of losing a male partner for refusing to engage in unprotected sex, especially when there is a low ratio of men to women [98], is often 'perceived' as more imminent than the health risk of contracting HIV. In many cases, the vital needs of the woman (i.e., safety, connection, true love) are in the $20 \%$. AA women seek coping strategies to tolerate the emptiness stemming from the $20 \%$ void. Often times, coping strategies include substance use, encouraging women to make sexual decisions that contradict their internal compass (i.e., having condomless sex with a partner women perceive as unfaithful). Intervention strategies aimed at dispelling myths associated with minimizing the sexual act and associated risks along with rewriting sexual scripts are needed to change the communication norms for this population by supporting ways to use effective communication that doesn't jeopardize the relationship instead of suppressing the emotional needs in the $20 \%$, which supports the need of a coping strategy (i.e., substance use). Without such strategies, the disproportionate burden of HIV disease to YSSAAW will persist.

We explored whether parental dynamics was a key factor in the development of sexual scripts and communication norms. Across the majority of descriptions on parental dynamics was the fear of a parent learning of the sexual debut of these women, most of who debuted during their teen years. Transcripts suggest that fear of parents learning of their sexual debut encouraged them to secretly engage in sexual activities. Alternatively, when YSSAAW had an established open communication style with their parents and they felt safe to discuss their sexual debut, they reported benefitting from this communication style and felt safety in the social support offered by the parent. The open communication style between parent and child has the potential for substantial benefit; however, this was found in the minority of this sample. Absence of a father-daughter relationship was also disclosed. Lack of protection that stems from the father's absence may contribute to sexual script development where women seek to fill the love of a father through sex with a male partner. This coping strategy contributes to acceptance of partner infidelity [46], as the presence of a male figure is valued more highly than the adoption of a sexual script that may lead to his absence.

Study findings contribute to the literary conversation on the importance of condomless sex in HIV transmission. Condomless sex was a part of the sexual script that was routinely reported by YSSAAW in this sample, including those with a STI history. A disconnect between the perception of HIV risk versus actual risk was apparent. In some cases, women felt at risk for STIs due to their partner's infidelity, but did not feel at risk for HIV. This dissociative expression of risk may also be a part of the coping strategy that encourages YSSAAW to sustain the relationship with male partners amidst perceptions of partner infidelity. In other cases, women with a STI history reported adoption of preventive strategies, including routine HIV/STI testing, in addition to other components of a comprehensive HIV prevention strategy. The prevalence of condomless sex with main and casual partners in this sample is alarming given that unprotected vaginal intercourse is the most common mode of HIV infection worldwide [99]. The inseparable link between longevity and condomless sex poses a challenge to HIV interventionists aiming to drive down HIV incidence among AA women. As stated earlier, prevention strategies must include rewriting of sexual scripts using culturally relevant theories, specifically the SST and the TGP, in the adaptation of interventions with established efficacy in diverse populations for utility and tailoring towards the needs of YSSAAW. 


\section{Conclusion}

YSSAAW recruited from a Houston-based public ED would benefit from clinical trials as behavioral interventions that address sexual health. The logic model found through the interviews demonstrate that emotional wounds and norms inform decision making, which informs sexual scripts that determine whether these women adopt preventive or high risk sexual practices. This model requires the attention of clinicians and scientists, who inform HIV prevention through clinical and research practice, to identify and address sexual scripts aligned with high risk sexual practices among minority women in the US who are most vulnerable to HIV/STI infections.

\section{Limitations}

The primary limitation of this exploratory study was the relatively small sample size $(\mathrm{N}=30)$; thus, findings cannot be generalized to a broader population of YSSAAW. YSSAAW interviewed were involved in diverse types of sexual relationships; thus, findings cannot be applied to subgroups by marital status (i.e., single, living with partner, married). This pilot study should be replicated with a larger sample size and at other emergency departments. A secondary study limitation is that the data was based on self-report. This exploratory study is a first step towards providing descriptive information on predictors of sexual scripts for an understudied population of AA women that would benefit from intervention.

\section{Future Research}

We identified potential empowerment strategies that stemmed from interview findings. Predictors of sexual scripts identified through the interviews will be used by the authorship team to guide the adaptation of HIV interventions with established efficacy for utility in future interventions for YSSAAW and other populations at significant risk for HIV. As an example, the qualitative data in the interview transcripts will be used as real-life case-scenarios and the situations presented will be used for role-playing. This data will be used to increase other YSSAAW's perceived vulnerability to HIV, as we expect the scenarios presented in the interviews among YSSAAW will be relatable to other YSSAAW presenting to the emergency department for care. A grant proposal to the National Institute of Health is underway to explore utility of these interviews as a mechanism to help women identify the gaps between their perceptions of HIV risk versus their actual HIV risk.

More research is needed to examine the influence of substance use on condomless sex among YSSAAW. This matter requires the attention of behavioral researchers and should be incorporated in comprehensive HIV prevention strategies targeting vulnerable populations, specifically minority women. YSSAAW would benefit from the adoption of a more comprehensive HIV prevention strategy that not only utilizes routine HIV testing and monogamy, but also includes pre-exposure prophylaxis and supports consistent condom use. Through HIV prevention strategies, YSSAAW will be encouraged to increase intentions to avoid substance use during sexual activity. YSSAAW require interventions that provide information and tools that align with their cultural values and beliefs. Tailored interventions are capable of averting future HIV/ STI infections, an evitable outcome when high risk sexual practices persist in the absence of intervention. In order for interventions with established efficacy in diverse populations to establish efficacy among YSSAAW with sexual scripts that encourage risk sexual behaviors, they must be adapted in a way to embrace the culture of YSSAAW (free of judgment), improve their self-worth and esteem to value their own sexual health, and increase their perceptions of HIV/STI as a real and personal threat. Previous researchers found that low perceptions of HIV/STI risk is the most important factor to be addressed through HIV prevention among Latino and AA women [3]. The interview findings presented are the initial steps towards building a program that will increase perceived vulnerability to HIV by directly addressing cultural appropriateness and sexual scripts through the adaptation of existing evidence based interventions for utility among YSSAAW.

\section{Acknowledgement}

Manuscript conceptualization was supported by a career development award from the American Psychological Association R25MH83635.

\section{References}

1. Center for Disease Control and Prevention (CDC) (2015) Diagnoses of HIV infection in the United States and dependent areas.

2. Center for Disease Control and Prevention (CDC) (2016) HIV among women.

3. Roberts ST, Kennedy BL (2006) Why are young college women not using condoms? Their perceived risk, drug use and developmental vulnerability may provide important clues to sexual risk. Arch Psychiatr Nurs 20: 32-40.

4. Hoxhaj S, Davila JA, Modi P, Kachalia N, Malone K, et al. (2011) Using nonrapid HIV technology for routine, opt-out HIV screening in a high-volume urban emergency department. Ann Emerg Med 58: S79-S84.

5. Maxwell JC (2014) Substance abuse trends in Texas. J Proceedings of the Community Epidemiology Work Group, Austin, Texas 42.

6. Center for Disease Control and Prevention (CDC) (2015) Pre-exposure prophylaxis (PrEP).

7. Haukoos JS, Mehta SD, Harvey L, Calderon Y, Rothman RE (2009) Research priorities for human immunodeficiency virus and sexually transmitted infections surveillance, screening and intervention in emergency departments Consensus-based recommendations. Acad Emerg Med 16: 1096-1102.

8. Monroe KW, Weiss HL, Jones M, Hook EW (2003) Acceptability of urine screening for Neisseria gonorrheae and Chlamydia trachomatis in adolescents at an urban emergency department. Sex Transm Dis 30: 850-853.

9. Bachmann LH, Pigott D, Desmond R, Jones M, Lumpkins J, et al. (2003) Prevalence and factors associated with gonorrhea and chlamydial infection in at-risk females presenting to an urban emergency department. Sex Transm Dis 30: 335-339.

10. Todd CS, Haase C, Stoner BP (2001) Emergency department screening for asymptomatic sexually transmitted infections. Am J Public Health 91: 461-464.

11. Mehta SD, Rompalo A, Rothman RE, Londner MS, Zenilman JM (2003) Generalizability of STD screening in urban emergency departments: Comparison of results from inner city and urban sites in Baltimore, Maryland Sex Transm Dis 30: 143-148.

12. Mehta SD, Rothman RE, Kelen GD, Quinn TC, Zenilman JM (2001) Unsuspected gonorrhea and chlamydia in patients of an urban adult emergency department: a critical population for STD control intervention. Sex Transm Dis 28: 33-39.

13. Begley CE, Vojvodic RW, Seo M, Burau K (2006) Emergency room use and access to primary care: Evidence from Houston, Texas. J Health Care Poor Underserved 17: 610-624.

14. Brown ER, Ojeda VD, Wyn R, Levan R (2000) Racial and ethnic disparities in access to health insurance and health care. UCLA Center for Health Policy Research and Kaiser Family Foundation: Los Angeles, California.

15. Center for Disease Control and Prevention (CDC) (2015) Effective interventions: HIV prevention that works.

16. Crepaz N, Marshall KJ, Aupont LW, Jacobs ED, Mizuno Y, et al. (2009) The efficacy of HIVISTI behavioral interventions for African American females in the United States: A meta-analysis. Am J Public Health 99: 2069-2078.

17. Lyons MS, Raab DL, Lindsell CJ, Trott AT, Fichtenbaum CJ (2007) A novel emergency department based prevention intervention program for people living with HIV: Evaluation of early experiences. BMC Health Serv Res 7: 164.

18. American College of Emergency Physicians (2007) HIV testing and screening in the emergency department. Ann Emerg Med 50: 209.

19. Arbelaez C, Block B, Losina E, Wright EA, Reichmann WM, et al. (2009) Rapid 
HIV testing program implementation: lessons from the emergency department. Int J Emerg Med 2: 187-194.

20. Batey DS, Hogan VL, Cantor R, Hamlin CM, Ross-Davis K, et al. (2012) Shor communication routine HIV testing in the emergency department: Assessment of patient perceptions. AIDS Res Hum Retroviruses 28: 352-356.

21. Bonney EA, Crosby R, Odenat L (2004) Repeat HIV testing among lowincome minority women: A descriptive analysis of factors influencing decisional balance. Ethn Dis 14: 330-335.

22. Centers for Disease Control and Prevention (CDC) (2007) Rapid HIV testing in emergency departments--three U.S. sites, January 2005-March 2006. MMWR Morb Mortal Wkly Rep 56: 597-601.

23. Arbelaez C, Wright EA, Losina E, Millen JC, Kimmel S, et al. (2012) Emergency provider attitudes and barriers to universal HIV testing in the emergency department. J Emerg Med 42: 7-14

24. Brown J, Shesser R, Simon G, Bahn M, Czarnogorski M, et al. (2007) Routine HIV screening in the emergency department using the new US centers for disease control and prevention guidelines: Results from a high-prevalence area. J Acquir Immune Defic Syndr 46: 395-401.

25. Branson BM, Handsfield HH, Lampe MA, Janssen RS, Taylor AW, et al. (2006) Revised recommendations for HIV testing of adults, adolescents and pregnant women in health-care settings. MMWR Recomm Rep 55:1-17.

26. Haukoos JS (2012) The impact of nontargeted HIV screening in emergency departments and the on-going need for targeted strategies. Arch Intern Med 172: $20-22$.

27. McCarthy JJ, Green PJ, McNeese-Ward MI, Roberts MJ (2010) A novel approach to emergency department HIV Testing - Conventional Testing Rapidly: 427 New Positives And 55,529 Tests In 16 Months. Society of Academic Emergency Medicine 2010 Annual Meeting: Phoeniz, AZ.

28. McCarthy JJ, Green PJ, Roberts MJ, McNeese-Ward ML (2010) Making "Routine" routine: A novel approach to HIV testing in the emergency department. Society of Academic Emergency Medicine 2010 Annual Meeting: Phoeniz, AZ.

29. New York State Department of Health (2013) Important health advisory: Emergency department interventions to prevent opioid overdose. New York State.

30. DiClemente RJ, Murray C, Graham T, Still J (2015) Overcoming barriers to HPV vaccination: A randomized clinical trial of a culturally-tailored, media intervention among African American girls. Hum Vaccin Immunother 11: 2883-2894.

31. Hill MJ (2010) An APP to HPV: Using a Smartphone application to encourage HPV catch-up vaccine uptake among young AA Women (Part 1) C.f.t.P.o.H.S.a.t.U.o.T. Health, Editor, Houston.

32. Hill MJ, Hu N, Granado M, Durrett A (2014) An assessment of sexual risk among HIV negative women tested in an emergency department setting. 142nd American Public Health Association Conference.

33. Hill M, Okugo G (2014) Emergency Medicine Physician Attitudes towards HPV vaccine uptake in an emergency department setting. Hum Vaccin Immunother 10: 2551-2556

34. Hill M, Grimes R (2014) A pilot study assessing whether African American women visiting the emergency department give different answers than an anonymous, internet-based population. Emergency Medicine and Health Care 5.

35. Hill M, Granado M, Peters R, Markham C, Ross M, et al. (2013) A pilot study on the use of a smartphone application to encourage emergency department patients to access preventive services: Human papillomavirus vaccine as an example. Emergency Medicine and Health Care 1.

36. Boutin-Foster C, McLaughlin N, Gray A, Ogedegbe A, Hageman I, et al. (2010) Reducing HIV and AIDS through Prevention (RHAP): A theoretically based approach for teaching HIV prevention to adolescents through an exploration of popular music. J Urban Health 87: 440-451.

37. Jones S, Hostler H (2002) Sexual script theory: An integrative exploration of the possibilities and limits of sexual self-definition. J Psychol Theol 30: 120-130.

38. Kim JL, Sorsoli CL, Collins K, Zylbergold BA, Schooler D, et al. (2007) From sex to sexuality: Exposing the heterosexual script on primetime network television. J Sex Res 44: 145-157.

39. Parsons JT, Vicioso KJ, Punzalan JC, Halkitis PN, Kutnick A, et al. (2004) The impact of alcohol use on the sexual scripts of HIV-positive men who have sex with men. J Sex Res 41: 160-172.
40. Reed D, Weinberg MS (1984) Premarital coitus: Developing and established sexual scripts. Soc Psychol Q 47: 129-138.

41. Tolman DL, Kim JL, Schooler D, Sorsoli CL (2007) Rethinking the associations between television viewing and adolescent sexuality development: Bringing gender into focus. J Adolesc Health 40: e9-e16.

42. Stokes C (2007) Representin' in cyberspace: Sexual scripts, self-definition and hip hop culture in black American adolescent girls' home pages. Cult Health Sex 9: 169-184.

43. DePadilla L, Windle M, Wingood G, Cooper H, DiClemente R (2011) Condom use among young women: Modeling the theory of gender and power. Health Psychol 30: 310-319

44. Wingood GM, DiClemente RJ (1992) Cultural, gender, and psychosocia influences on HIV-related behavior of African-American female adolescents: Implications for the development of tailored prevention programs. Ethn Dis 2 : 381-388.

45. Wingood GM, DiClemente RJ (2000) Application of the theory of gender and power to examine HIV-related exposures, risk factors and effective interventions for women. Health Educ Behav 27: 539-565.

46. Wingood GM, DiClemente RJ (1998) Partner influences and gender-related factors associated with non-condom use among young adult African American women. Am J Community Psychol 26: 29-51.

47. Albarracín D, Johnson BT, Fishbein M, Muellerleile PA (2001) Theories of reasoned action and planned behavior as models of condom use: A metaanalysis. Psychol Bull 127: 142-161.

48. Hennessy M, Bleakley A, Fishbein M, Brown L, Diclemente R (2010) Differentiating between precursor and control variables when analyzing reasoned action theories. AIDS Behav 14: 225-236.

49. Jones R (2006) Sex scripts and power: a framework to explain urban women's HIV sexual risk with male partners. Nurs Clin North Am 41: 425-436, vii.

50. Wiederman M (2005) The gendered nature of sexual scripts. Fam J 13: 496-502.

51. McLellan-Lemal E, Toledo L, O'Daniels C, Villar-Loubet O, Simpson C, et al. (2013) "A man's gonna do what a man wants to do": African American and Hispanic women's perceptions about heterosexual relationships: A qualitative study. BMC Womens Health 13: 27

52. Ross JN, Coleman NM (2011) Gold digger or video girl: The salience of an emerging hip-hop sexual script. Cult Health Sex 13: 157-171.

53. Jones R, Oliver M (2007) Young urban women's patterns of unprotected sex with men engaging in HIV risk behaviors. AIDS Behav 11: 812-821.

54. Hussen SA, Bowleg L, Sangaramoorthy T, Malebranche DJ (2012) Parents, peers and pornography: The influence of formative sexual scripts on adult HIV sexual risk behaviour among Black men in the USA. Cult Health Sex 14: 863-877.

55. Holman A, Sillars A (2012) Talk about "hooking up": The influence of college student social networks on non-relationship sex. Health Commun 27: 205-216.

56. Vannier SA, O'Sullivan LF (2011) Communicating interest in sex: Verba and nonverbal initiation of sexual activity in young adults' romantic dating relationships. Arch Sex Behav 40: 961-969.

57. Erickson PI, Badiane L, Singer M (2013) The social context and meaning of virginity loss among African American and Puerto Rican young adults in Hartford. Med Anthropol Q 27: 313-29.

58. Robinson BB, Bockting WO, Rosser BR, Miner M, Coleman E (2002) The sexual health model: Application of a sexological approach to HIV prevention. Health Educ Res 17: 43-57.

59. Bowleg L, Lucas KJ, Tschann JM (2004) "The ball was always in his court": An exploratory analysis of relationship scripts, sexual scripts and condom use among African American women. Psychol Women Q 28: 70-82.

60. Roye CF, Tolman DL, Snowden F (2013) Heterosexual anal intercourse among Black and Latino adolescents and young adults: A poorly understood high-risk behavior. J Sex Res 50: 715-722.

61. Gagnon JH, Simon W (1973) Sexual conduct: The social sources of human sexuality. Aldine, Chicago, IL.

62. Singer JL, Salovey P (1991) Organized knowledge structure and personality: Person schemas, self-schemas, prototypes, and scripts. Person schemas and maladaptive interpersonal patterns, U.o.C. Press, Chicago 33-79. 
Citation: Hill MJ, Granado M, Villarreal Y, Fuega J, Robinson DJ, et al. (2017) Predictors of Sexual Scripts Among Young, Sexually-Active, SubstanceUsing African American Women. J AIDS Clin Res 8: 655. doi: 10.4172/2155-6113.1000655

63. Hill M, Granado M, Stotts A (2016) Theoretical implications of gender, power and sexual scripts for HIV prevention programs aimed at young, substanceusing African-American women. J Racial Ethn Health Disparities.

64. Martyn KK, Hutchinson SA (2001) Low-income African American adolescents who avoid pregnancy: Tough girls who rewrite negative scripts. Qual Health Res 11: 238-256.

65. Pulerwitz J, Amaro H, De Jong W, Gortmaker SL, Rudd R (2002) Relationship power, condom use and HIV risk among women in the USA. AIDS Care 14: 789-800.

66. Wingood GM, Scd, DiClemente RJ (2000) Application of the theory of gender and power to examine HIV-related exposures, risk factors and effective interventions for women. Health Educ Behav 27: 539-565.

67. Amaro H (1995) Love, sex and power: Considering women's realities in HIV prevention. Am Psychol 50: 437-447.

68. Amaro H, Raj A, Reed E (2001) Women's Sexual health: The need for feminist analyses in public health in the decade of behavior. Psychol Women Q 25: 324-334

69. Bowleg L (2004) Love, sex and masculinity in sociocultural context. Men Masc 7: 166.

70. Fullilove RE, Fullilove MT, Bowser BP, Gross SA (1990) Risk of sexually transmitted disease among black adolescent crack users in Oakland and San Francisco, Calif. JAMA 263: 851-855.

71. Wingood GM, DiClemente RJ, Bernhardt JM, Harrington K, Davies SL, et al. (2003) A prospective study of exposure to rap music videos and African American female adolescents' health. Am J Public Health 93: 437-439.

72. Hill M, Granado M, Stotts A (2016) Theoretical implications of gender, power, and sexual scripts for HIV prevention programs aimed at young, substanceusing African American women. J Racial Ethn Health Disparities.

73. Dworkin SL, Beckford ST, Ehrhardt AA (2007) Sexual scripts of women: a longitudinal analysis of participants in a gender-specific HIV/STD prevention intervention. Arch Sex Behav 36: 269-279.

74. Dworkin SL, O'Sullivan L (2005) Actual versus desired initiation patterns among a sample of college men: Tapping disjunctures within traditional male sexual scripts. J Sex Res 42: 150-158.

75. Emmers-Sommer Allen M (2005) Safer sex in personal relationships: The role of sexual scripts in HIV infection and prevention. Lawrence Erlbaum Associates, Mahwah.

76. Harrison A et al (2006) Gender role and relationship norms among young adults in South Africa: Measuring the context of masculinity and HIV risk. J Urban Health 83: 709-722.

77. Hynie M, Lydon JE, Cote S, Wiener S (1998) Relational sexual scripts and women's condom use: The importance of internalized norms. J Sex Res 35: 370-380.

78. Kreager DA, Staff J, Gauthier R, Lefkowitz ES, Feinberg ME (2016) The double standard at sexual debut: Gender, sexual behavior and adolescent peer acceptance. Sex Roles 75: 377-392.

79. Trinh SL, Ward LM (2016) The nature and impact of gendered patterns of peer sexual communications among heterosexual emerging adults. J Sex Res 53: 298-308.

80. Gesselman AN, GD Webster, JR Garcia (2017) Has virginity lost its virtue? Relationship stigma associated with being a sexually inexperienced adult. J Sex Res 54: 202-213.

81. Hust SJ, Rodgers SB, Ebreo S, Stefani W (2016) Rape myth acceptance, efficacy and heterosexual scripts in men's magazines: Factors associated with intentions to sexually coerce or intervene. J Interpers Violence.

82. French BH, Neville HA (2016) What is non-consensual sex? Young women identify sources of coerced sex. Violence Against Women.
83. Littleton HL, Dodd JC (2016) Violent attacks and damaged victims: An exploration of the rape scripts of European American and African American U.S. College Women. Violence Against Women.

84. Coleman MN, Butler EO, Long AM, Fisher FD (2016) In and out of love with hip-hop: Saliency of sexual scripts for young adult African American women in hip-hop and Black-oriented television. Cult Health Sex 18: 1165-1179.

85. Leiting KA, Yeater EA (2017) A qualitative analysis of the effects of victimization history and sexual attitudes on women's hypothetical sexual assault scripts. Violence Against Women 23: 46-66.

86. Santo SD (2015) Why this secret twist on the $80 / 20$ rule makes relationships happier. Your Tango.

87. Hotton AL, French AL, Hosek SG, Kendrick SR, Lemos D, et al (2015) Relationship dynamics and sexual risk reduction strategies among heterosexual young adults: A qualitative study of sexually transmitted infection clinic attendees at an Urban Chicago Health Center. AIDS Patient Care STDS 29: $668-674$

88. Velasquez M, Stotts A (2003) Substance abuse and dependence disorders in women: Handbook of counseling women. Sage Publications: Sage Publications, pp: 482-503.

89. McDuff DR, Solounias BL, RachBeisel J, Johnson JL (1994) Psychiatric consultation with substance abusers in early recovery. Am J Drug Alcohol Abuse 20: 287-299.

90. Bernal G (2006) Intervention development and cultural adaptation research with diverse families. Fam Process 45: 143-151.

91. Ingham R, Stone N (2015) Topics for in-depth Interviews and focus group discussions, in sexual and reproductive health. The Department of Reproductive Health and Research.

92. McHugh ML (2012) Interrater reliability: The kappa statistic. Biochem Med (Zagreb) 22: 276-282.

93. Robert Ranningan L (2016) Emotional wounding. Speaking of Feelings.

94. Gullette DL, Lyons MA (2006) Sensation seeking, self-esteem and unprotected sex in college students. J Assoc Nurses AIDS Care 17: 23-31.

95. Pulerwitz J, Barker G, Segundo M, Nasciemento M (2006) Promoting more gender-equitable norms and behaviors among young men as an HIVIAIDSprevention strategy. Horizons Research Summary.

96. Connell RW (1987) Gender and power: Society, the person and sexual politics Stanford University Press, Standford, CA.

97. Zukoski AP (2011) Exploring power and sexual decision making among young Latinos residing in rural communities. Womens Health Issues 21: 450-457.

98. Adimora AA, Schoenbach VJ, Doherty IA (2006) HIV and African Americans in the southern United States: Sexual networks and social context. Sex Transm Dis 33: S39-45.

99. Boily MC, Baggaley RF, Wang L, Masse B, White RG, et al (2009) Heterosexual risk of HIV-1 infection per sexual act: Systematic review and meta-analysis of observational studies. Lancet Infect Dis 9: 118-129. 